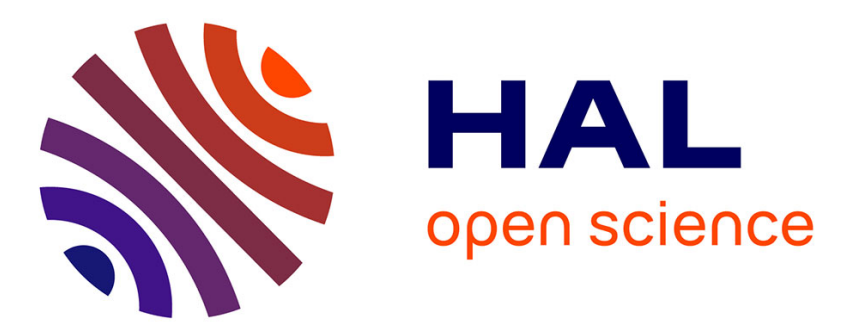

\title{
Introducing dynamic behavior of magnetic materials into a model of a switched reluctance motor drive
}

\author{
Fabien Sixdenier, Laurent Morel, Jean-Pierre Masson
}

\section{To cite this version:}

Fabien Sixdenier, Laurent Morel, Jean-Pierre Masson. Introducing dynamic behavior of magnetic materials into a model of a switched reluctance motor drive. IEEE Transactions on Magnetics, 2006, 42 (3), pp.398-404. 10.1109/TMAG.2005.862757 . hal-00141407

\section{HAL Id: hal-00141407 \\ https://hal.science/hal-00141407}

Submitted on 18 Apr 2007

HAL is a multi-disciplinary open access archive for the deposit and dissemination of scientific research documents, whether they are published or not. The documents may come from teaching and research institutions in France or abroad, or from public or private research centers.
L'archive ouverte pluridisciplinaire HAL, est destinée au dépôt et à la diffusion de documents scientifiques de niveau recherche, publiés ou non, émanant des établissements d'enseignement et de recherche français ou étrangers, des laboratoires publics ou privés. 


\title{
Introducing Dynamic Behavior of Magnetic Materials Into a Model of a Switched Reluctance Motor Drive
}

\author{
F. Sixdenier, L. Morel, and J. P. Masson \\ Centre de Génie Electrique de Lyon-UMR CNRS 5005, 69622 Villeurbanne cedex, France
}

\begin{abstract}
Dynamic hysteretic effects of magnetic materials are usually neglected in actuators modeling. In order to take into account these effects, we coupled a two-dimensional finite-element (FE) model in an original way with a magnetic equivalent circuit by using dynamic hysteretic flux tubes (DHFT). As an example of an application, we present the model of an ultrafast switched reluctance motor, in which the control of the power converter is of major importance, and where iron losses can reach critical values.
\end{abstract}

Index Terms_Eddy currents, finite-element method, magnetic hysteresis, reluctance motors.

\section{INTRODUCTION}

D YNAMIC hysteresis behavior in a magnetic core is usually neglected in actuators modeling. In some cases, the time variations of the magnetic flux density inside the actuator may be very fast (i.e., high-speed machines, machine fed by an inverter, etc.). Thus, the magnetic losses increase [1]-[5] and the usual electric signals are deformed. This deformation has an effect on mechanical quantities (i.e., torque), and may result in poor speed or torque control. It is difficult to take into account the magnetic material (hysteresis, eddy-current) laws by using a "pure" two-dimensional finite-element (2-D FE) simulation, owing to the high computational cost (CPU time and memory). In this paper, we propose a hybrid method, where a dynamic hysteresis flux tubes network [6], [7] is coupled to a 2-D FE simulation [8]. This method is an interesting alternative to classical FE methods for actuators modeling and industrial design, in that it allows to predict at a moderate cost the electric and magnetic quantities waveforms of a system composed by an actuator and its dedicated inverter. The 2-D FE method is used to describe the flux density distribution in strong gradients areas (notably in proximity of air gaps) where high precision is needed, whereas the flux tubes are used to describe the complex hysteretic magnetic phenomena (static and dynamic) inside the different magnetic parts of the actuator. The high permeability of the materials allows to approximate the detailed geometry of these tubes by a simple magnetic circuit (i.e., the leakage field outside the actuator is neglected). As an example of an application, we present an ultrahigh-speed double saliency switched reluctance motor (SRM) drive [9], [10] designed by the Laboratoire de genie industriel et productique de Metz (LGIPM). This SRM is a three-phase machine including six stator poles and two rotor poles (SRM 6/2). The nominal features of this machine are $200000 \mathrm{rpm}$ and $1 \mathrm{~kW}$ on the mechanical shaft. This machine is dedicated for high-speed applications, for the following reasons:

- the rotor dissipation is reduced;

- the high torque-to-weight and power-to-weight ratios;

- the winding and the power electronics converter are simplified.

Digital Object Identifier 10.1109/TMAG.2005.862757

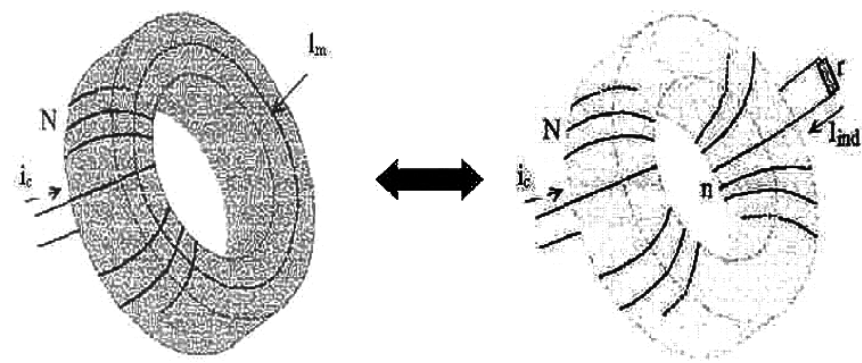

Fig. 1. Equivalence between a conductive core with eddy currents and an insulated core with a fictitious coil supplying a resistance.

The paper is organized as follows. First, the dynamic hysteretic flux tubes (DHFT) and the FE model are described. The coupling between these two models is presented in the next section; the inverter and the mechanical behavior of the system are taken into account as well. Finally, the main results are presented.

\section{DynAmic Hysteretic Flux Tubes}

Let us consider a conductive magnetic core, with a constant cross section $S_{\mathrm{ec}}$ and a mean length $l_{\mathrm{m}}$, excited by a coil of $N$ turns where a current $i_{\mathrm{c}}$ is circulating. The dynamic hysteresis includes two main phenomena:

— macroscopic eddy currents;

- dynamic behavior due to wall motion.

In the dynamic hysteretic flux tube method, this core is modeled by an insulating lossless core, and a fictitious secondary coil of $n$ turns linked to a resistance $r$ (Fig. 1).

The model postulates [4] the equivalence between the two dynamic effects, and the magnetomotive force localized in the fictitious secondary coil.

By applying Ampere's law to the insulated core, one obtains

$$
\oint_{l_{m}} \mathbf{H}_{\mathrm{sta}} \cdot d l=N \cdot i_{c}+n \cdot I_{\text {ind }}
$$

$\mathbf{H}_{\text {sta }}$ is the magnetic excitation field. If the field is homogeneous in the core, one may write

$$
H_{\text {sta }} \cdot l_{m}=N \cdot i_{c}+n \cdot I_{\text {ind }}
$$




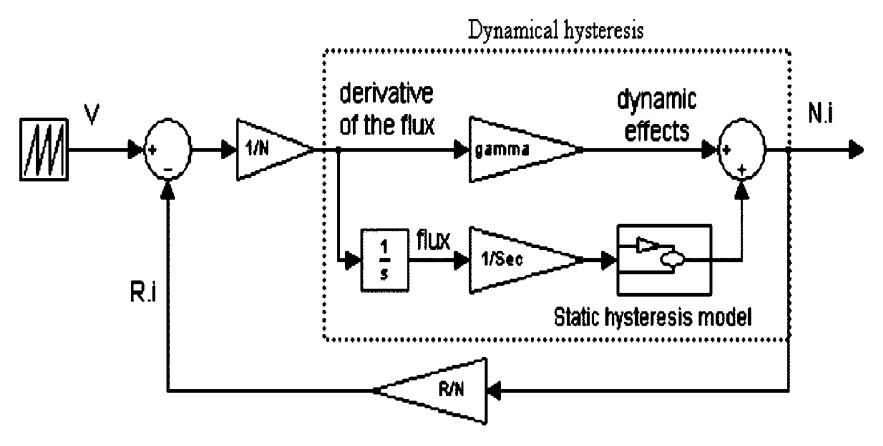

Fig. 2. Example of the links between the electric and magnetic quantities.

$I_{\text {ind }}$ is the induced current in the fictitious coil

$$
I_{\text {ind }}=-\frac{n}{r} \cdot \frac{d \phi}{d t}
$$

$\phi$ is the instantaneous flux in the core cross section. Thus, the governing equation representing the evolution of the state of the core is

$$
N \cdot i_{c}-l_{m .} F^{-1}\left[\frac{\phi}{S_{\mathrm{ec}}}\right]=\gamma \frac{d \phi}{d t}
$$

where $\gamma=n^{2} / r$. The second term globally represents the dynamic effects inside the core. Neither the number of turns, $n$ of the fictitious coil nor the value of the resistance $r$ can be evaluated separately. Only the term $\gamma$ can be identified by comparing with a measured dynamic hysteresis loop with a simulated one. The function $F^{-1}$ returns the magnetic excitation field $H_{\text {sta }}$ for a given value of $\phi$, by taking into account the magnetization history. Indeed, $F^{-1}$ is a static hysteresis model, which allows to describe nonsymmetrical minor loops. When the primary coil is supplied by a voltage source $V$ with a resistor $R$ in series, the electric equation is

$$
V=R \cdot i_{c}+N \cdot \frac{d \phi}{d t}
$$

By replacing the term $i_{\mathrm{c}}$ by its value given by (4), it becomes

$$
V=N \cdot \frac{d \phi}{d t}\left(1+\frac{\gamma R}{N^{2}}\right)+\frac{R}{N} l_{m} \cdot F^{-1}\left(\frac{\phi}{S_{\mathrm{ec}}}\right) .
$$

Thus, the model of a magnetic core fed by a voltage source can be represented by the diagram in Fig. 2 .

The result is a description in the time domain of the magnetomotive force as a function of the derivative of the flux $\phi$ applied to a flux tube element. Several dynamic flux tubes can be linked to form an equivalent magnetic circuit.

\section{Finite-ElemENT Model}

As only a few parts of the magnetic circuit are meshed, the dynamic behavior of the material inside the FE domain may be neglected. Thus, a classical 2-D magnetostatic formulation [11] is used:

$$
-\nabla \cdot\left(\frac{1}{\mu} \nabla \cdot \mathbf{A}\right)=\mathbf{J}_{z}
$$

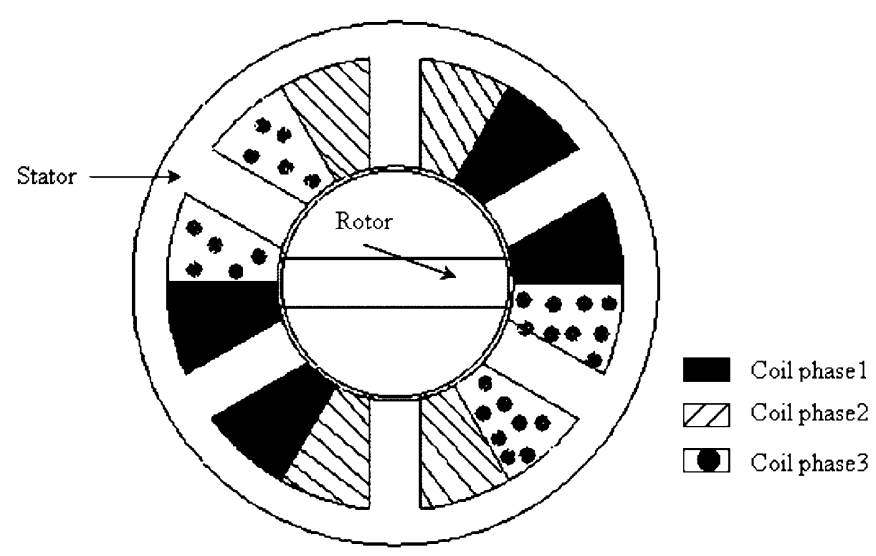

Fig. 3. Geometry of the $6 / 2$ switched reluctance motor drive.

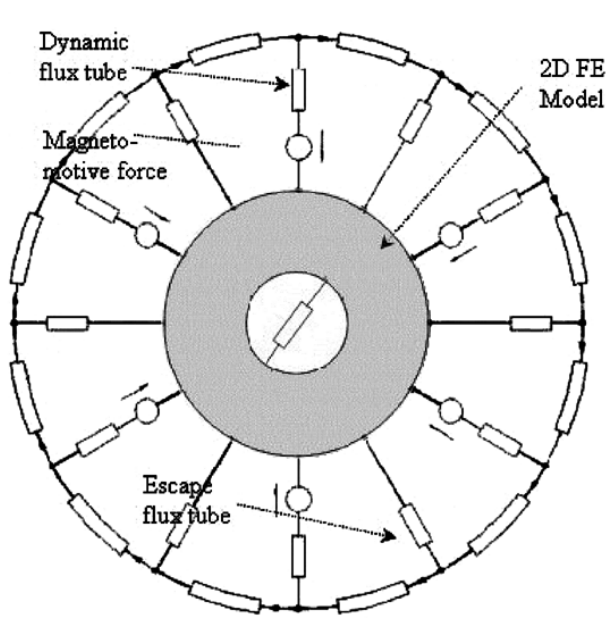

Fig. 4. Association of a flux tube network and a FE model (in gray).

$\mathbf{J}_{z}(x, y)$ is the source current density, and $\mathbf{A}$ is the magnetic vector potential. However, as only part of the actuator is actually meshed, special boundary conditions must be imposed in order to ensure the coupling with the flux tubes network.

\section{COUPLing OF THE DifFerent Models}

For the sake of simplicity, the geometry of the actuator taken as an example is represented in Fig. 3. The SRM has six stator teeth, two rotor teeth, and consequently three phases.

\section{A. Coupling FE and Flux Tubes}

Fig. 4 shows a representation of the linkage of the FE model with the DHFT model. As anticipated in the introduction, the FE model provides a fine representation of the region surrounding the air gap, whereas the DHFT model is used for the stator and the rotor. Remark that the FE domain has to be meshed at each time step, owing to the motion of the rotor.

Assume that one coil of the actuator is fed by a voltage source, and that the value $E_{C}(t)$ of the voltage across this coil is known at any time. The linked flux can be obtained by integrating this voltage: $\phi(t)=\left(1 / N_{C}\right) \int_{-\infty}^{t} E_{C}\left(t^{\prime}\right) d t^{\prime}$, where $N_{C}$ is the number of turns of the coil. The coupling between this coil and the FE domain is implemented by imposing that: 


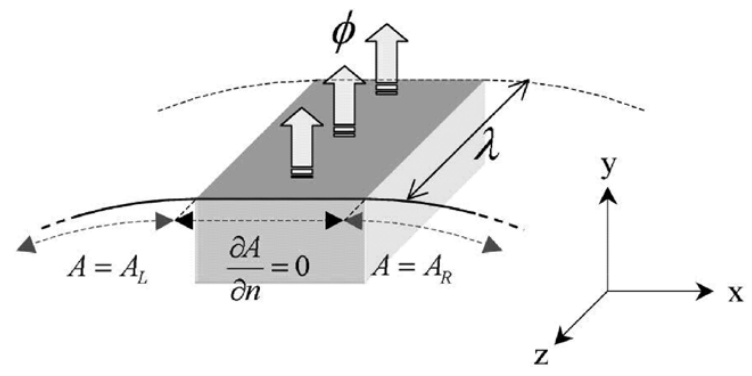

Fig. 5. Illustration of defining a flux with two magnetic vector potentials.

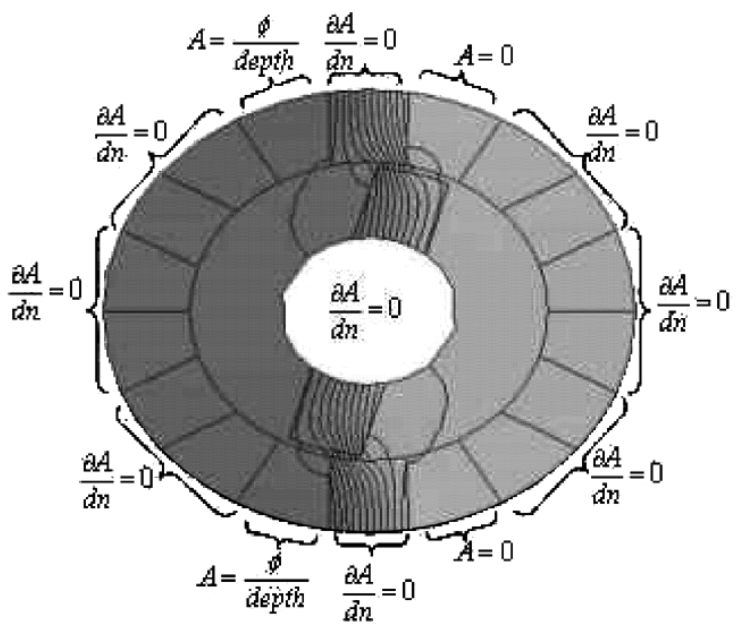

Fig. 6. Boundary conditions and plot of the equipotential lines of the magnetic vector potential A for the 2-D FE model of the air gap and its surroundings.

1) the flux density is perpendicular to the tooth cross section at the boundary of the FE domain;

2) the flux $\phi$ is conserved.

The first condition is imposed by using homogeneous Neumann conditions $(\partial A / \partial n)=0$ at the cross section of the tooth, and two different nonhomogeneous Dirichlet conditions on the left side $\left(A=A_{L}\right)$ and right side $\left(A=A_{R}\right)$ of the tooth (see Fig. 5). The flux conservation is imposed at any time by setting the difference between $A_{L}$ and $A_{R}$, that is

$$
A_{L}-A_{R}=\frac{\phi(t)}{\lambda}
$$

where $\lambda$ is the depth of the system [11].

Fig. 6 represents an example of FE computation, together with the proper boundary conditions.

The Neumann conditions, imposed onto the boundaries which correspond to tooth, may be considered as "flux gates" [12], [13]. On the other hand, the Dirichlet boundary conditions can be considered as "flux walls." One observes that the vector potential of one of these flux walls may be arbitrarily set to zero, in that only the difference between two potential is physically meaningful.

One wonders how to choose the extension of the FE domain, in order to obtain reliable and accurate results- that is, which part of the actuator really needs to be accurately represented through a FE model. Indeed, we have checked that the results are stable over a large range of the width of the FE domain; in particular, the magnetostatic energy in the air gap does not depend upon the extension of the FE domain (more details will be given in a future paper).

Let $[Z]$ be the topology matrix, that is: $Z_{i j}=+1(-1)$ when the flux through the $i$ th branch enter (exit) into the $j$ th node, or 0 otherwise (the orientation of each branch can be chosen arbitrarily). The law of the flux conservation in each node can be written using the matrix formalism as $[Z] \cdot[\phi]=0$, where the vector $[\phi]$ contains the flux through all the tubes. The vector $[\phi]$ can be separated into two distinct parts: $[\phi]=\left[\phi_{1} \phi_{2}\right]$, where $\phi_{1}$ contains the flux through the branches linked to the FE model (flux gates), and $\phi_{2}$ contains the fluxes in the stator head. The flux conservation can be expressed by

$$
\left[\begin{array}{ll}
Z_{1} & Z_{2}
\end{array}\right] \cdot\left[\begin{array}{l}
\phi_{1} \\
\phi_{2}
\end{array}\right]=0
$$

Thus, $\phi_{2}$ can be easily deduced from $\phi_{1}$ by using (8). When all the fluxes are known, the differences of magnetic potential of each flux tube are then also known by using (4). The difference of magnetic potential in the FE domain is calculated through the equation

$$
n i_{\mathrm{FE}}=\left.\frac{\partial W_{\mathrm{em}}}{\partial \phi}\right|_{\theta_{m}=\mathrm{cte}}
$$

where $W_{\mathrm{em}}$ is the electromagnetic energy inside the FE domain, $\phi$ is the imposed flux, and $\theta_{m}$ is the mechanical position of the rotor. All these differences of magnetic potential will be useful for the coupling of this hybrid model with the electric circuit equations.

\section{B. Coupling With an Electrical Circuit}

As it can be seen in Fig. 2, the model needs the total magnetomotive force due to the flux, and its derivative. Indeed, this magnetomotive force comes from Ampere's law

$$
\oint \vec{H} \cdot d \vec{l}=N \cdot i_{c} \text {. }
$$

The magnetomotive difference of potential given by the FE domain and some others given by the stator head and teeth (stator and rotor) flux tubes are summed in order to give the total magnetomotive force $N \cdot i_{c}$ created by the phase flux imposed to the system (Fig. 7). This total magnetomotive force is then multiplied by the gain $R / N$ and returned to the electric circuit ( $R$ is the value of the phase resistance, and $N$ is the number of turns of the coil). Thus, the model can be fed by any voltage source and/or inverter.

\section{Validation on a Simple Test Device}

In order to test the validity of the coupling, a dedicated device [8] has been constructed. This device is a U core where an air gap is present like that shown in Fig. 8.

The hysteresis loop present in the material of the device is measured using a sensor coil and a gaussmeter and compared with the one obtained by simulation.

The measured and simulated hysteresis loops are in a good agreement that allows predicting iron losses Fig. 9. 


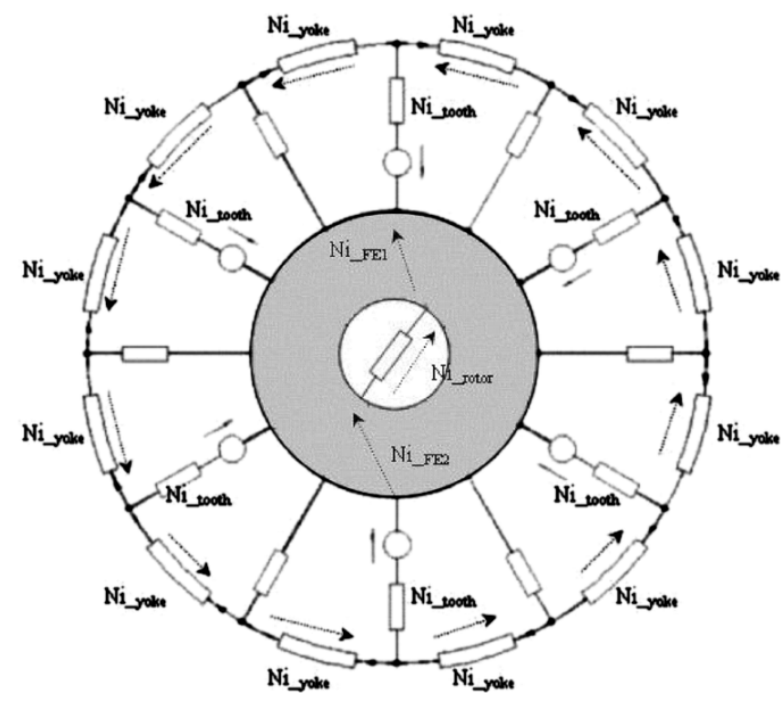

Fig. 7. Representation of the magnetomotive differences of potential of the FE zone and of each ferromagnetic flux tube.

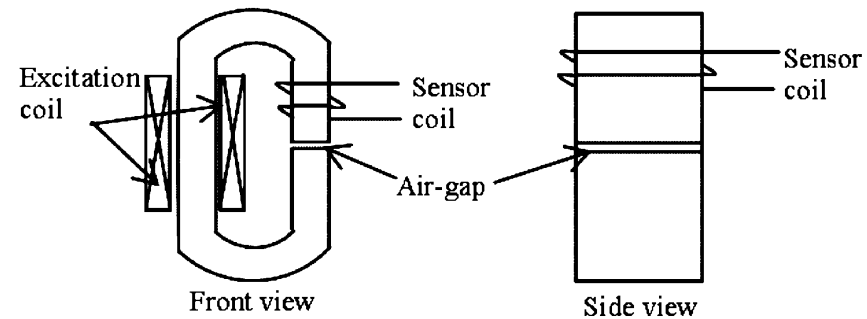

Fig. 8. Geometry of the test device.

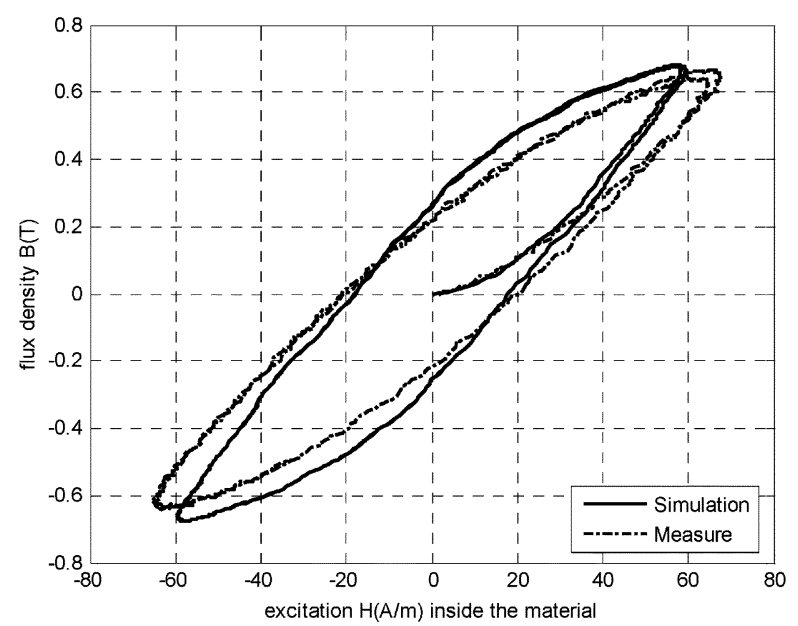

Fig. 9. Simulated and measured hysteresis loops inside the magnetic material.

\section{Modeling of a Simplified Inverter}

Usually, the inverter dedicated to this type of machine is an asymmetrical half bridge (Fig. 10), because the current is unidirectional. Also, this inverter uses a current and position control law: a coil of the SRM must be fed while the rotor is between two distinct positions, and the current should not exceed a certain value to avoid overheating —or, at worst, destroying the coil. Thus, between two distinct rotor positions, the coil is supplied with a switching current regulation. With this type of inverter, there are only three values of voltages applied to a coil:
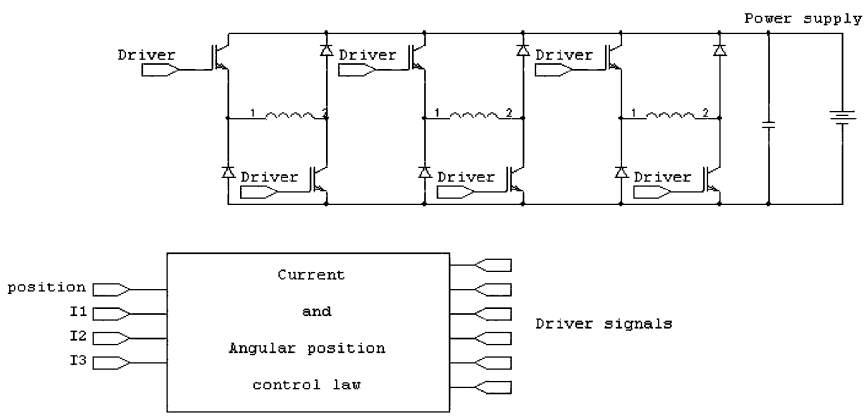

Fig. 10. Asymmetrical half bridge for a three-phase machine with a current and position control law.

$E, 0$, or $-E$, depending on the rotor position and on the current value ( $E=$ power supply voltage). The voltage source seen in Fig. 2 is replaced by a programmable voltage source, function of the rotor position and of the phase current considered (this programmable source can take the three values: $E, 0$, or $-E$ ).

\section{E. Mechanical Coupling}

The mechanical magnitudes of acceleration, speed, position, and torque has been simulated by using the virtual works method:

$$
\begin{aligned}
T_{\mathrm{em}} & =-\left.\frac{\partial W_{\mathrm{em}}}{\partial \theta_{m}}\right|_{\phi=c t e} \\
T_{\mathrm{em}}-T_{r} & =J \frac{d \Omega}{d t}+f \Omega
\end{aligned}
$$

where $T_{\mathrm{em}}$ is the electromagnetic torque, $T_{r}$ is the load torque, $J$ is the moment of inertia, $f$ is the viscous rubbing coefficient, $\Omega$ is the angular speed, and $\theta_{0}$ is the initial rotor position.

It is worth remarking that by using this description of the system, three physical domains (mechanic, electric, and magnetic) are strongly coupled.

\section{RESULTS}

The model has been implemented by using the software MATLAB/SIMULINK, with the partial derivative equations (PDE) toolbox to simulate the 2-D FE domain. First, some results (voltages, currents, and torque) obtained by the model are be compared to those computed with the FE software FLUX2D in the magneto evolutionary mode-in this case all the geometry is meshed, in that at present time no measurements on the real device are available. Then, the flux density versus the excitation field in one tooth is presented for transient and steady-state operation.

\section{A. Integral Magnitudes for Transient and Steady States}

The results presented here have been obtained for an initial speed of $5000 \mathrm{rpm}$ and $25000 \mathrm{rpm}$ at no-load conditions.

One observes (Fig. 11) that the currents computed by both our model and FLUX2D are very similar at the beginning of the simulation. Conversely, when the current starts to decrease, the currents computed by our model vanish after those computed by FLUX2D. One may argue that this discrepancy between the computations is due to the different material model implemented in FLUX2D. 


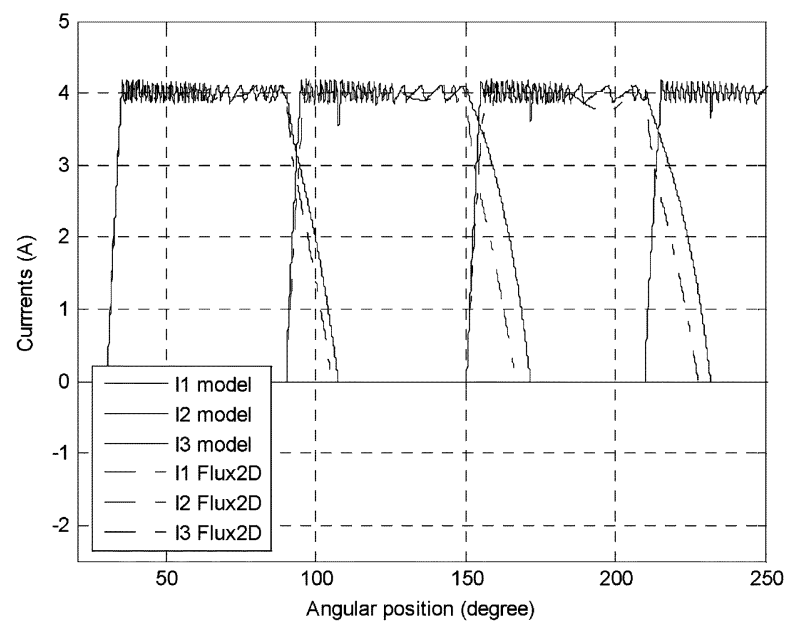

Fig. 11. Currents of the phases computed by FLUX2D (dashed lines) and by the model (solid lines) for an initial angular speed of $5000 \mathrm{rpm}$.

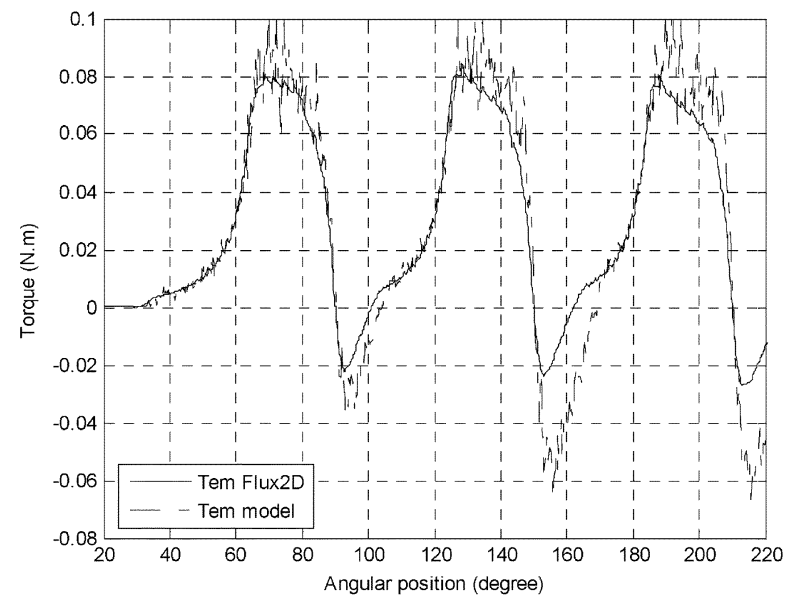

Fig. 12. Comparison of the torque versus angular position computed by FLUX2D (dashed line) and by the model (solid line).

This discrepancy has an effect on the computed torque (Fig. 12): indeed, as a consequence of the currents' delay, the time interval where the torque is negative is longer in the case of our model.

Fig. 11 clearly shows that the machine is accelerating. This shows that the model can handle transient states very easily.

Fig. 11 also shows that at the beginning of the simulation the results obtained with our model and FLUX2D are very close. Conversely, at the end of the simulation the speed computed by our hybrid model is lower than the one obtained with FLuX2D. This is related to the discrepancy observed for the negative part of the torque. For the same conditions of power supply and the same parameters of the control law, the machine stops accelerating when the speed is about $25000 \mathrm{rpm}$; as a consequence, the currents' waveforms change.

It can be observed (Fig. 14) that the dynamic behavior of the magnetic material clearly influences the currents' waveforms. As the speed is increasing, the eddy currents become more and more important and the behavior of the material is far from reversible operation. The influence of these electric waveforms

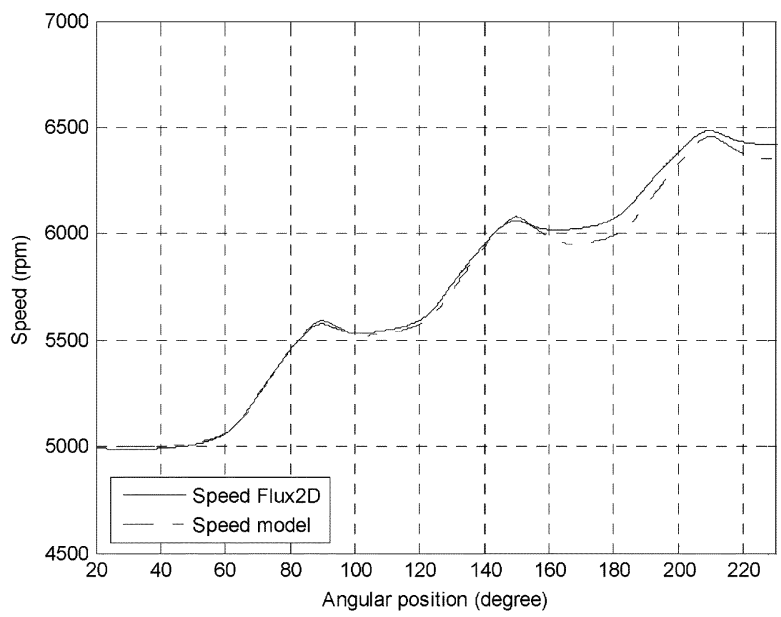

Fig. 13. Comparison of the speed versus angular position of the model and FLUX2D.

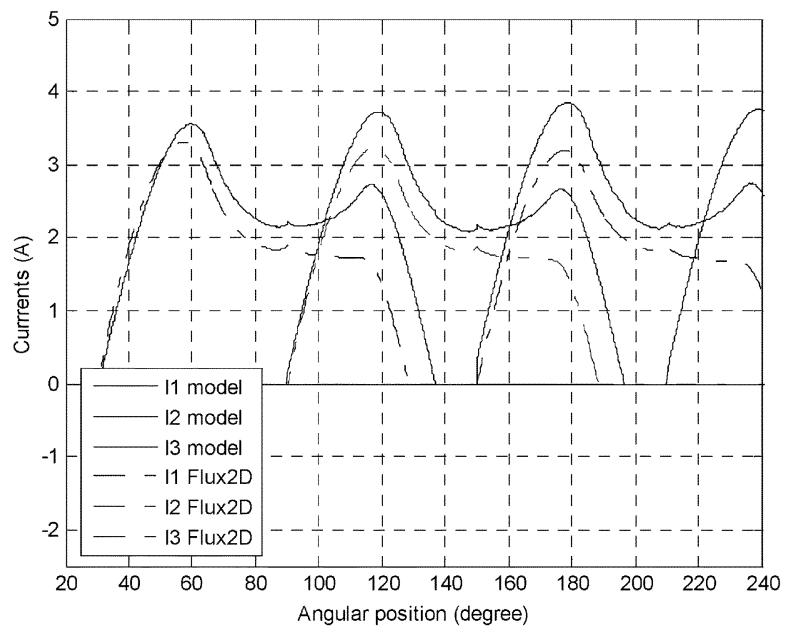

Fig. 14. Currents of the phases computed by FLUX2D (dashed lines) and by the model (solid lines) for a quasi-constant speed of $25000 \mathrm{rpm}$.

changes on the mechanical quantities can be observed by comparing Figs. 12 and 15 respectively to Figs. 11 and 14.

The differences between the results obtained with our model and those obtained with FLUX2D can be explained by the same arguments. The material behavior lowers the theoretical performances obtained with the initial control law. A modified control law could allow obtaining a better mechanical behavior.

\section{B. Local Quantities Useful for Loss Prediction}

As the model describes the dynamic behavior of the magnetic parts of the machine, we are able to know flux density versus excitation field loops for both tests seen before. We have plotted the mean flux density versus the mean excitation field loop inside the tooth stator (Fig. 17) and in the yoke (Fig. 18).

It can be observed that for $n_{0}=5000 \mathrm{rpm}$, the flux density inside the tooth becomes very saturated. Moreover, some minor loops can be observed; these loops are a consequence of the current regulation. Conversely, for $n_{0}=25000 \mathrm{rpm}$ the current never exceeds the (maximum) limit, so no minor loops are observed, and the flux density inside the tooth is lower than in the former case $\left(n_{0}=5000 \mathrm{rpm}\right)$. 


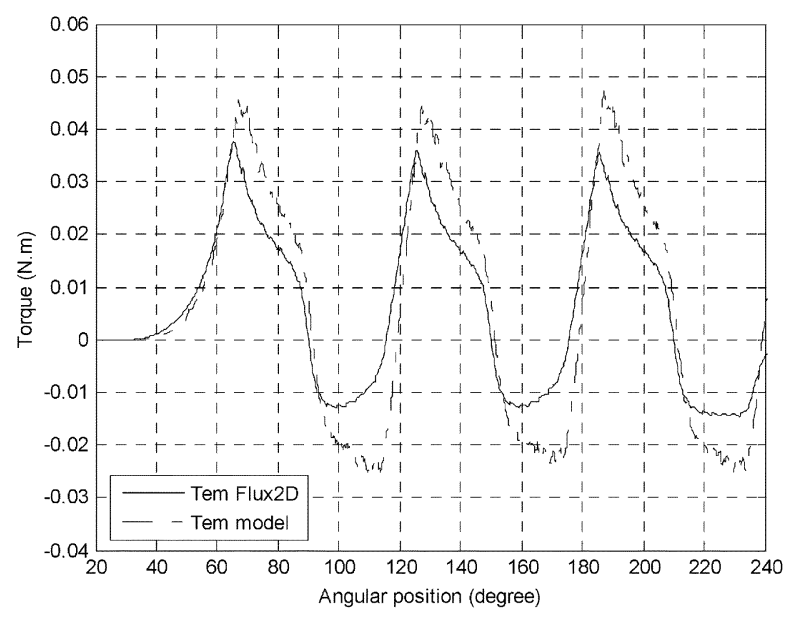

Fig. 15. Comparison of the torque versus angular position given by FLUX2D and the model at quasi-constant speed.

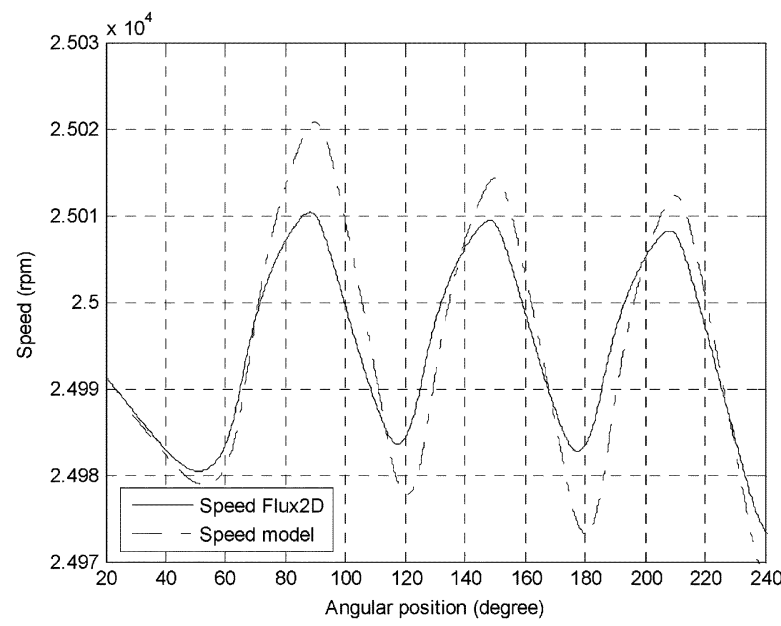

Fig. 16. Comparison of the speed versus angular position of the model and FLUX2D at quasi-constant speed.

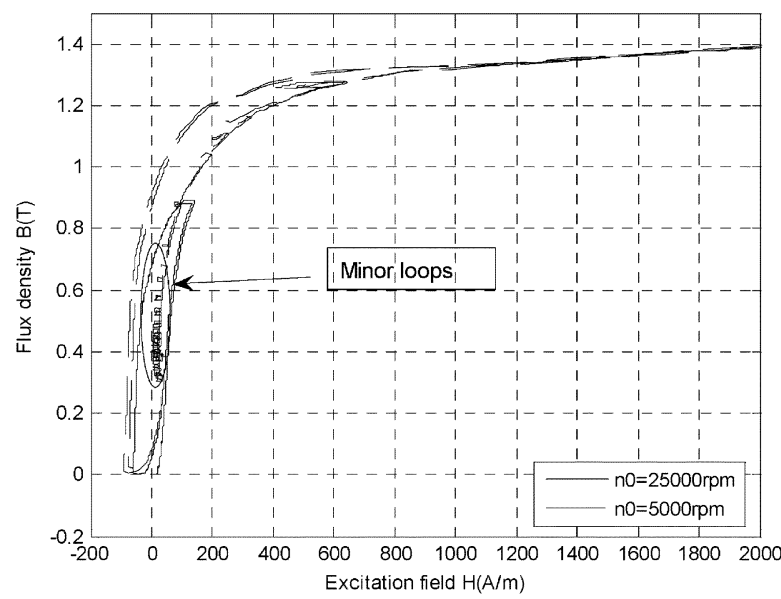

Fig. 17. Induction field versus excitation field inside a stator tooth loop for the test $n_{0}=5000 \mathrm{rpm}$ and $n_{0}=25000 \mathrm{rpm}$.

\section{Power Balance}

When the simulation is finished, a power balance can be done. Fig. 19 shows a power balance with respect to the mechanical speed at no-load conditions.

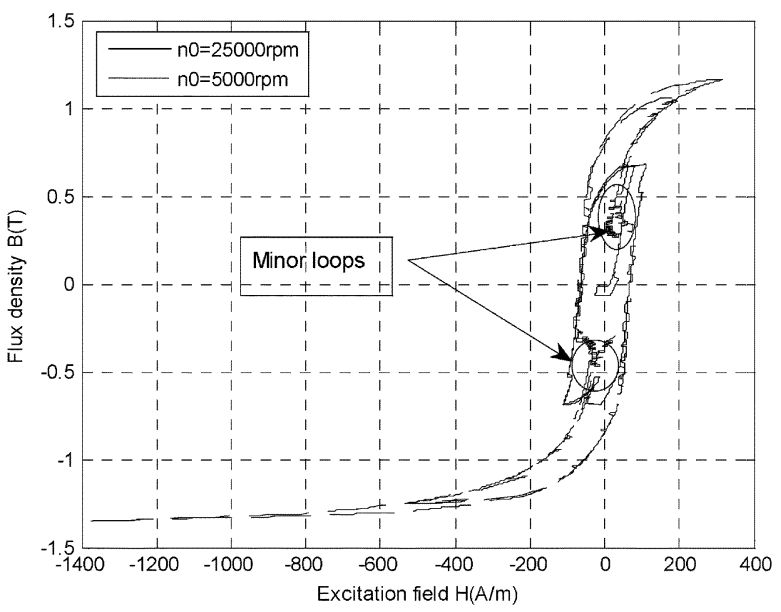

Fig. 18. Mean induction field versus excitation field inside the yoke loop for the tests $n_{0}=5000 \mathrm{rpm}$ and $n_{0}=25000 \mathrm{rpm}$.

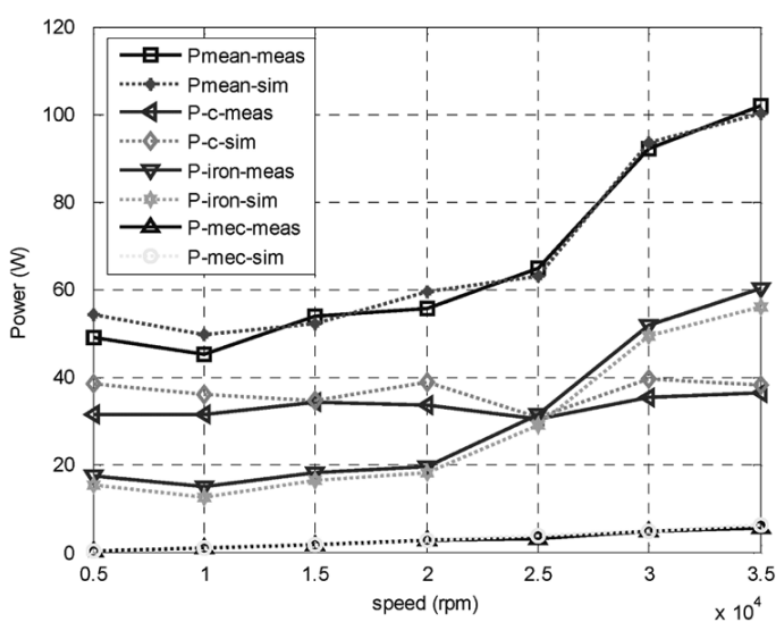

Fig. 19. Power balance versus mechanical speed.

The terms "Pmean," "P-c," "P-iron," and "P-mec" denote the mean electric power, the copper losses, the iron losses, and the mechanical losses, respectively. The suffixes "meas" and "sim" denote measured and simulated, respectively. The results given by the simulation are in good agreement with those that are measured. Fig. 19 clearly shows that when the mechanical speed exceeds $25000 \mathrm{rpm}$, the iron losses exceed the copper losses. This shows that for some kind of actuators (high-speed ones) the iron losses cannot be neglected. Then, dedicated models with a low computation time have to be created in order to take these considerations into account.

\section{CONCLUSION}

A 2-D FE model has been coupled to a magnetic equivalent circuit made with dynamic hysteretic flux tubes. This model can be fed by a simplified inverter to simulate the whole system, both in transient and harmonic regime. The results have shown that, at low frequencies, the electric, magnetic, and mechanic quantities computed by our model are very close to those obtained by the commercial software FLUX2D. When the frequency of the supply increases, the magnetic material behavior has more and 
more influence on all the quantities; this fact justifies the introduction of material laws into the simulation model. The model can also predict the hysteresis loops in every flux tube, for any kind of supply.

Future developments of this model could allow predicting the iron losses with the model. For this first approach, the $35000 \mathrm{rpm}$ speed corresponds to the higher limit for the assumptions of the dynamic global flux tube model [4]: a more accurate description including dynamic hysteretic diffusion of the magnetic field [14], [15] has to be found for higher frequencies. This model would possibly allow studying accurately the control law, in order to maximize mean torque on the mechanical shaft.

\section{ACKNOWLEDGMENT}

The authors would like to thank all the members of the LGIPM (Laboratoire de genie industriel et productique de Metz, Université de Metz) for the data needed for this study.

\section{REFERENCES}

[1] L. R. Dupré, R. Van Keer, and J. A. A. Melkebeek, "A computational model for the iron losses in rotating electrical machines," Int. J. Eng. Sci., no. 36, pp. 699-709, 1998.

[2] O. Bottauscio, A. Canova, M. Chiampi, and M. Repetto, "Rotational hysteresis and eddy current losses in electrical motor stators under nonconventional supply," J. Magn. Magn. Mater, vol. 254-255, pp. 241-243, 2003.

[3] T. Chevalier, A. Kadous-Lebouc, B. Cornut, and C. Cester, "A new dynamic hysteresis model for electrical steel sheet," Physica B, vol. 275, pp. 197-201, 2000.
[4] F. Marthouret, J. P. Masson, and H. Fraisse, "Modeling of a non-linear conductive magnetic circuit," IEEE Trans. Magn., vol. 31, no. 6, pp. 4065-4067, Nov. 1995.

[5] J. F. Lee, J. C. Kim, and D. S. Hyun, "Hysteresis modeling of synchronous reluctance motor considering PWM input voltage," Physica B: Condens. Matter, vol. 275, no. 1-3, pp. 238-247, Jan. 2000.

[6] V. Ostovic, Dynamics of Saturated Electric Machines. Berlin, Germany: Springer-Verlag, 1989.

[7] J. Turowski, M. Turowski, and M. Kopec, "Method of three dimensional network solution of leakage field of three-phase transformers.," IEEE Trans. Magn., vol. 26, no. 5, pp. 2911-2919, Sep. 1990.

[8] F. Sixdenier, B. Ducharne, L. Morel, and J. P. Masson, "Coupled programming: Finite elements-Dynamical hysteresis flux tubes for nonhomogeneous circuit modeling," in Compumag 2003, vol. III. Saratoga Springs, NY, Jul. 13-17, 2003.

[9] L. Morel, H. Fayard, R. Vives-Fos, A. Galindo, and G. Abba, "Study of ultra high speed switched reluctances motor drive," in IEEE-IAS 2000, vol. 1, Rome, Italy, Oct. 2000, pp. 87-92.

[10] L. Morel, H. Fayard, and R. Vives-Fos, "A new rotor solution for a high speed machine," in ICEM 2000, vol. 2/3, Finland, pp. 664-668.

[11] M. V. K. Chari and S. J. Salon, Numerical Methods in Electromagnetism. New York: Academic, 2000.

[12] J. Gyselink, L. Vandevelde, and J. Melkebeek, "Coupling finite elements and magnetic and electrical networks in magnetodynamics," in ICEM98, vol. 2/3, Istanbul, Turkey, Sep. 2-4, 1998.

[13] H. Gerserm, K. Hameyer, and T. Weiland, "Field-circuit coupled models in electromagnetic simulation," J. Comput. Appl. Math., vol. 168, pp. 125-133, 2004.

[14] M. A. Raulet, B. Ducharne, J. P. Masson, and G. Bayada, "The magnetic field diffusion equation including dynamic hysteresis: A linear formulation of the problem," IEEE Trans. Magn., vol. 40, no. 2, pp. 872-875, Mar. 2004.

[15] K. B. Tatis, A. G. Kladas, and J. A. Tegopoulos, "Harmonic iron loss determination in laminated iron cores by using a particular 3-D finiteelement model," IEEE Trans. Magn., vol. 40, no. 2, pp. 860-863, Mar. 2004.

Manuscript received April 14, 2005; revised November 23, 2005 (e-mail: sixdenier@cegely.univ-lyon1.fr). 